\title{
A MODIFIED METHOD FOR THE 1000-COMPARTMENT FLUCTUATION TEST
}

\author{
E. S. LEUNG \\ Department of Biology, University of Saskatchewan, Soskatoon, Saskatchewan, \\ Canada S7N OWO
}

Received 14.iii.74

A VERY sensitive method for the determination of enhanced spontaneous mutation rates in yeast has been developed by von Borstel and his collaborators $(1971,1973)$. Mutation rates are calculated from the zeroth term of a Poisson distribution, as first applied by Luria and Delbrück (1943). The method is limited to the measurement of revertant frequencies from auxotrophy to prototrophy. Selection pressure is imposed onto the growing cultures when the limiting amino acid is depleted from the medium, thus only prototrophic revertants will form colonies.

Our attempt was to compare spontaneous mutation rates, during aerobic and anaerobic growth, from antibiotic sensitivity to resistance in a certain mutant yeast strain, where the wild type was resistant to the level of antibiotic specified (Rank and Bech-Hansen, 1973). Therefore, it was necessary to modify the procedure so that selection pressure could be placed upon the cultures after cell growth. The principle was essentially the same as detailed in the references given above, hence only alterations were reported in this paper.

The tray with the compartments was made by drilling wells into a piece of clear, autoclavable, plastic material $2 \mathrm{~cm}$ thick. Each compartment could hold approximately $3 \mathrm{ml}$. The tray was placed in a box to keep out contaminants and to prevent evaporation of medium. Disposable plastic trays with wells of various sizes used in tissue culture might be plausible alternatives.

The culture medium was prepared as follows: 1 per cent yeast extract, 2 per cent bacto-peptone, $0 \cdot 1$ per cent glucose and 0.1 per cent agar (Difco). A fermentable carbon source was used because the determination of mutation rates during anaerobic growth was part of the programme. Agar was added to immobilise the cells. This medium was quite fluid at room temperature, thus allowed even distribution of cells by a magnetic stirrer. The batch was mass inoculated to give a final concentration of 3000 cells per ml. Aliquots of $1 \mathrm{ml}$ size were then dispensed into the compartments. The cells stopped dividing when the carbon source was depleted (four days at $25^{\circ}$ C.). Minute colonies were observed evenly suspended in the medium from top to bottom (fig. 1). The size of the inoculum and the amount of glucose to be added depend on the mutation rate studied, and thus have to be determined for the individual experiment concerned.

The cell population had to be determined after the period of limited growth. It was found that the agar medium did not facilitate the use of a hemocytometer in counting cells, since a much greater volume was trapped under the cover glass. It was possible to harvest the cells by centrifugation and have them resuspended in water, but the loss of a small number of cells 
during the process seemed unavoidable. An alternative would be serial dilutions and plating. There was no difficulty in diluting the cultures with water after growth for several days.

To visualise the number of mutational events that had occurred in the compartments, selection pressure (presence of an antibiotic) and a new supply of energy source were added to the medium. The antibiotic and glycerol in a $0 \cdot 1$ per cent agar base were layered on to the cultures (Glycerol was used as carbon source, because resistance to an antibiotic is defined as the ability to grow in the presence of that antibiotic using a non-fermentable carbon source). If this was done carefully, the immobilised minute colonies would stay undisturbed (fig. 1). Higher concentrations of agar were tried and found to be workable up to 0.2 per cent. Colonies tended to take the form of " needles" at concentrations lower than $0 \cdot 1$ per cent.

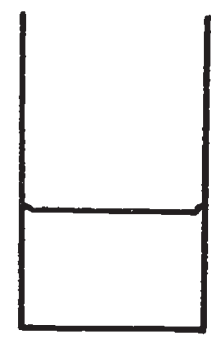

1

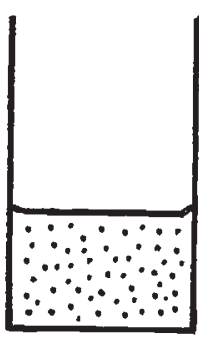

2

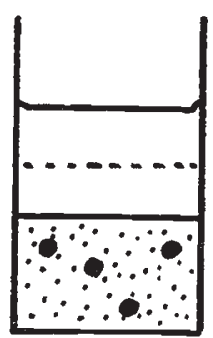

3

FIG. 1.-Diagrammatic representation of the cross-sectional view of a compartment of the fluctuation test. (1) One-millilitre culture with 3000 cells. (2) Pin-point colonies after 4 days. (3) Mutational events visualised as large, solid colonies.

In approximately 10 days after the above treatment, solid resistant colonies were observed in 10 to 30 per cent of the compartments, while the minute colonies had also increased a little in size. Resistance was confirmed by picking the colonies with a Pasteur pipette and streaking them on to an antibiotic supplemented medium. Similarly, the minute colonies were shown to be sensitive to the drug. Cell counts from mutant-free compartments showed that the sensitive cells had undergone one or two doublings before growth was inhibited by the antibiotic. Thus it was deemed necessary to add the antibiotic one or two days before the energy source was made available to the cells. More time had to be allowed for the diffusion of antibiotic throughout the medium if anaerobic growth was involved, since this step had to be done in the cold.

In conclusion, the chief finding presented in this paper is the use of thin agar in the 1000-compartment fluctuation test. Compared with von Borstel's method, the above procedure is more time-consuming and requires greater care. However, it might be found applicable to studies where spontaneous mutation rates of diverse nature are to be sought. It seems probable that the method can also be adapted for other microorganisms such as bacteria.

Acknowledgments.- The author wishes to thank Dr G. H. Rank for provision and for initiating the experimental study. Thanks are also extended to $\mathrm{Dr}$ N. T. Bech-Hansen for suggestions concerning the apparatus used, and to $\mathrm{Mr} \mathrm{S}$. Woytowich for making them. 


\title{
REFERENGES
}

LURIA, S. E., AND DELBRÜCK, M. 1943. Mutations of bacteria from virus sensitivity to virus resistance. Genetics, 28, 491-511.

RANK, G. H., AND BEGH-HANSEN, N. T. 1973. Single nuclear gene inherited cross resistance and collateral sensitivity to 17 inhibitors of mitochondrial function in S. cerevisiae. Molec. gen. Genet., 126, 93-102.

VON BORSTEL, R. C., CAIN, K. T., AND STEINBERG, G. M. 1971. Inheritance of spontaneous mutability in yeast. Genetics, 69, 17-27.

VON BORSTEL, R. C., QUAH, S.-K., STEINBERG, C. M., FLURY, F., AND GotTLIEB, D. J. C. 1973. Mutants of yeast with enhanced spontaneous mutation rates. Genetics Supplement, 73, 141-151.

\section{STABILITY OF DIPLOID CLONES OF THE CELLULAR SLIME MOULD DICTYOSTELIUM DISCOIDEUM}

\author{
E. B. GINGOLD* \\ Department of Biochemistry, School of Biological Sciences, University of Leicester, \\ Leicester $L E I$ TRH
}

Received 24.iv.74

\begin{abstract}
Summary
Haploid cultures of Dictyostelium discoideum obtained from the axenic strain $\mathrm{Ax}-2$ were fused with each other and also with the non-axenic strain X9 to produce diploid clones. The diploids were heterozygous for two recessive drug resistance genes and thus by measuring the segregation under nonselective conditions of haploids expressing drug resistance, a measure of the frequency of haploidisation was obtained. It was demonstrated that $\mathrm{X} 9$ when fused with an axenic strain produced diploids of greater stability than those obtained by the fusion of two axenic strains. It has been postulated that X9 carries a gene (or a series of genes) that give rise to diploid stability
\end{abstract}

\section{Introduction}

The cellular slime mould Dictyostelium discoideum has a complex life-cycle (Bonner, 1959). In the presence of a food supply this organism grows as single celled amoebae. Thus when plated on to a bacterial lawn amoebal clones ingest the bacteria and clear plaques in the lawn similar at first in appearance to the plaques formed by phage. On exhaustion of the food supply however the amoebae aggregate and differentiate into spore and stalk cells-these fruiting bodies appearing in the centre of the plaque.

The system has proven to be attractive for studies of cell differentiation (Garrod and Ashworth, 1973). However the development of a useful genetic system has been far behind the cell biological and biochemical studies. By examination of chromosome number (Ross, 1960) and correlation of this with spore size and shape (Sussman and Sussman, 1962) it was shown that $D$. discoideum, although generally found as a haploid, can also exist in a diploid state. However, the nature of the conversions between these states remained obscure until evidence was found for a parasexual

* Present address: Department of Biology, Hatfield Polytechnic, Hatfield, Herts. 33/2-s2 